\title{
Validación de un sistema inteligente de recomendación híbrida en federaciones de repositorios de Objetos de Aprendizaje
}

Paula Rodríguez Universidad Nacional de Colombia parodriguezma@unal.edu.co Néstor Duque Méndez Universidad Nacional de Colombia ndduqueme@unal.edu.co

Demetrio Ovalle Universidad Nacional de Colombia dovalle@unal.edu.co

Valentina Tabares Universidad Nacional de Colombia vtabaresm@unal.edu.co

\section{Resumen}

En la actualidad los objetos de aprendizaje OA, que son recursos educativos cuya característica distintiva son los metadatos descriptivos, han tomado gran importancia para apoyar los procesos de enseñanza aprendizaje de estudiantes que cada día tienen acceso a más información; la dificultad al recuperar los OA se encuentra al especificar las palabras o cadena de consulta, para hacer las búsquedas y encontrar un recurso que se adapte a lo que se quiere encontrar y a sus características específicas. Es allí donde surgen los sistemas de recomendación, como apoyo a los usuarios a encontrar OA relevantes, en este artículo se realizan pruebas de funcionamiento de un sistema de recomendación híbrido aplicado a diferentes repositorios de OA y federaciones de repositorios, con el fin de validar su uso y pertinencia al momento de acceder a un recurso educativo.

\section{Palabras clave}

Federación de repositorios de objetos de aprendizaje, MAE, medida de precisión, relevancia, repositorios de objetos de aprendizaje, sistemas de recomendación,

\begin{abstract}
Currently learning objects LO are educational resources, whose main characteristic is descriptive metadata. They have become very important to support students teaching and learning processes, having a constant access to more information, the difficulty when retrieving LO is to specify words or query string to search and find an adaptive resource related to what is wanted to find and its specific characteristics. Hence, some recommendation systems emerge as user's support to find relevant LO. This paper deals with functional tests of a hybrid recommender system applied to different LO repositories and federations of repositories, in order to validate their use and relevance when accessing an educational resource.
\end{abstract}

Keyword

Federation of learning objects repositories, MAE, measurement accuracy, relevance, learning object repository, recommendation systems

\section{Introducción}

Actualmente con los grandes volúmenes de información digital disponibles, la computación de alta velocidad y la comunicación a través de redes ubicuas, permiten acceder y recuperar muchos recursos educativos (Peña, et. al., 2002). Entre ellos se encuentran los Objetos de Aprendizaje (OA), que se caracterizan por su disponibilidad inmediata a través de repositorios basados en web y por sus metadatos descriptivos que permiten su búsqueda y recuperación. Hacer búsquedas refinadas es difícil, incluso para usuarios avanzados (Wang et. al., 2007)(Mayorga et al., 2007), esta dificultad se puede atacar a través de los Sistemas de Recomendación SR, que surgen como aplicaciones que ofrecen sugerencias sobre un ítem a un usuario; esta sugerencia se obtiene a partir de sus características, preferencias anteriores o de las preferencias de una comunidad que tiene gustos y opiniones similares a la suya (Sanjuán et al., 2009). Un sistema de 
recomendación se puede distinguir de un sistema de recuperación de información por la personalización de sus respuestas a un usuario en particular (Burke, 2007). En este artículo se presenta la validación de un sistema de recomendación híbrido propuesto en (Rodríguez et al., 2013), aplicado a diferentes Repositorios de Objetos de Aprendizaje ROA; en el modelo se aplica una estrategia de integración para tres tipos de recomendación: Contenido, Colaborativa y Conocimiento.

La organización del artículo es la siguiente: en la sección 2, se expone el referente teórico. En la sección 3, se hace referencia a los principales trabajos relacionados con el modelo integral de recomendación propuesto, aplicado a ROA y se presenta el modelo de recomendación híbrido. En la sección 4, se presenta el método para aplicar las pruebas de validación; posteriormente en la sección 5, se presentan los resultados de la validación. Finalmente, se presentan las conclusiones y el trabajo futuro.

\section{Referente teórico}

A continuación se presentan los principales elementos que componen el sistema de recomendación híbrido aplicado a repositorios.

\subsection{Objetos de Aprendizaje (OA), Repositorios (ROA) y Federaciones}

Aunque que en la actualidad se aceptan diferentes definiciones para los OA, se puede concluir que un OA es cualquier entidad digital que parte de un diseño instruccional apropiado y que puede ser usado, reutilizado o referenciado durante un proceso de enseñanza-aprendizaje, con el objetivo de generar conocimientos, habilidades, actitudes y competencias en función de las necesidades del estudiante (Learning Technology Standards Committee, 2002)(Wiley, 2001). Adicionalmente los OA tienen metadatos descriptivos que identifican los recursos educativos y facilitan su búsqueda y recuperación desde diferentes fuentes, especificando la sintaxis y la semántica de los atributos necesarios para describirlo (Edwards et al., 2007). Entre los más importantes se encuentran el IEEE-LOM (Learning Technology Standards Committee, 2002), DublinCore (http://dublincore.org/) y OBAA (Vicari et al., 2009).

Los OA están contenidos en Repositorios de Objetos de Aprendizaje (ROA) que alojan múltiples tipos de recursos educativos y sus metadatos (Downes, 2001)(Tabares et al., 2012). Actualmente existen ROA reconocidos y utilizados a nivel mundial tales como: MERLOT $^{1}$, GATEWAY ${ }^{2}$ de EEUU y JORUM ${ }^{3}$ de Reino Unido, entre otros.

Una federación ofrece un enfoque unificado de representación de los repositorios (Tabares et al., 2012), sirve para facilitar la administración uniforme de aplicaciones para descubrir y acceder a los contenidos de los OA disponibles en un grupo de ROA. Entre las federaciones de ROA más conocidas se pueden citar las siguientes: FEB ${ }^{4}$ Federación de Educación de Brasil, ARIADNE ${ }^{5}$ que surge de un proyecto europeo, FROAC $^{6}$ que es la federación de repositorios de objetos de aprendizaje en Colombia (Tabares et al., 2012), entre otras.

\footnotetext{
${ }^{1}$ Multimedia Educational Resource for Learning and Online Teaching. Universidad Estatal de California (http://www.merlot.org/).

${ }^{2}$ Gateway toEducationalMaterials. Departamento de Educación de los EEUU. http://www.thegateway.org/

3 Service in Development in UK Further and Higher Education. Reino Unido.

${ }^{4}$ Federación de Educación de Brasil. Retrieved August 2011, from http://feb.ufrgs.br:8080/feb/

${ }^{5}$ Asociación europea abierta al mundo. http://ariadne.cs.kuleuven.be/AriadneFinder/

${ }^{6}$ Federación de Repositorios de Objetos de Aprendizaje Colombia

http://froac.manizales.unal.edu.co/froac/
} 


\subsection{Sistemas de Recomendación (SR)}

Los SR surgieron a mediados de la década de los 90 (Li, 2010) con el fin de brindarle a los usuarios aquellos resultados de búsquedas de información cercanos a sus necesidades (Casali et al., 2011). Un SR puede realizar predicciones a partir del hecho que a un usuario le guste o no cierto ítem al que podría acceder (Chesani, 2002). Los SR tienen la capacidad de identificar preferencias y sugerir ítems relevantes para cada usuario; para ello se necesita de perfiles que almacenen la información y las preferencias de cada usuario (Cazella et al., 2010).

Existen muchas técnicas de SR, cada una puede ser usada para diferentes enfoques y en diferentes contextos, de acuerdo a las necesidades (Hdioud et al., 2012), además necesitan gran cantidad de información sobre los usuarios e ítems a recomendar para entregar resultados de calidad, esto se logra con la caracterización de los estudiantes y la retroalimentación que se realice de las recomendaciones (Li, 2010), (Sanjuán et al., 2009). Así, para el caso de OA el SR debería ser capaz de recomendar OA adaptados a un estudiante específico. Burke clasifica los SR en (Burke, 2002):

- Sistemas de Recomendación Basados en el Contenido: realiza la recomendación con base a un perfil de usuario creado que almacena sus intereses, el proceso de recomendación se hace sobre las características presentes en los ítems.

- Sistemas de Recomendación Colaborativos: construyen la recomendación como una agregación estadística/probabilística de las preferencias de otros usuarios.

- Sistemas de Recomendación Basado en Conocimiento: hace recomendaciones según el historial de navegación de un usuario.

El enfoque híbrido, busca la unión entre varios enfoques de recomendación con el objetivo de completar sus mejores características y hacer mejores recomendaciones (Cazella et al., 2010)(Burke, 2002). Aunque este tipo de sistemas híbridos tienen grandes ventajas como entregar recomendaciones de alta calidad, su presencia en la Web es baja, debido a su complejo desarrollo que tiene en cuenta: la gestión de un modelo de usuario, los mecanismos para definir y clasificar la información, la definición de métodos y técnicas adaptativas y la definición de correspondencias entre los usuarios.

\section{Estado del Arte y Sistema a validar}

Este apartado se divide en dos partes, en la primera se presenta la revisión del estado del arte y en la secunda está el modelo propuesto que va a ser evaluado.

En los últimos años se ha avanzado en temas relacionados a los SR de OA y se continúa evolucionando para cumplir con los nuevos requisitos y entregar recursos adaptados (Kahn \& Wilensky, 2006). Por otro lado, los sistemas de recomendación en línea son ampliamente utilizados para apoyar a los usuarios a encontrar información pertinente (Niemann et al., 2010). En algunos casos se utilizan métricas de calidad para recuperar OA por técnicas de minerías de datos, trabajando sobre las preferencias de los estudiantes (Sabitha et al., 2012), en otros trabajos se realizan recomendaciones basadas en el usuario, usando las medidas de correlación de Pearson y la similitud por distancia euclidiana (Sicilia, et al., 2010). Primo et al. (2009) proponen recomendar contenido a los usuarios, buscando la similitud entre el perfil del tema y lo que recomendó a un usuario, además se tiene en cuenta el contexto en el que el usuario recibirá la recomendación; y García y Montoyo (2008) proponen un método para la gestión personalizada de OA ubicados en el ROA: Lor@server que consiste en entregar OA adecuados según las preferencias de un usuario en una situación dada, mediante la utilización de una ontología de dominio. Aunque todos los trabajos realizan recomendaciones, se encontró que no manejan recomendaciones híbridas por sus dificultades de implementación, además en los perfiles de los usuarios solo se tienen en cuenta sus preferencias. 


\section{Sistema híbrido de Recomendación de Objetos de Aprendizaje propuesto}

En (Rodríguez et al., 2013), se presentó el modelo integral de recomendación de OA que reúne varios enfoques de recomendación con el fin de obtener mejores resultados. En la figura 1 se puede ver el modelo propuesto. A continuación se explican los elementos que componen el modelo propuesto.

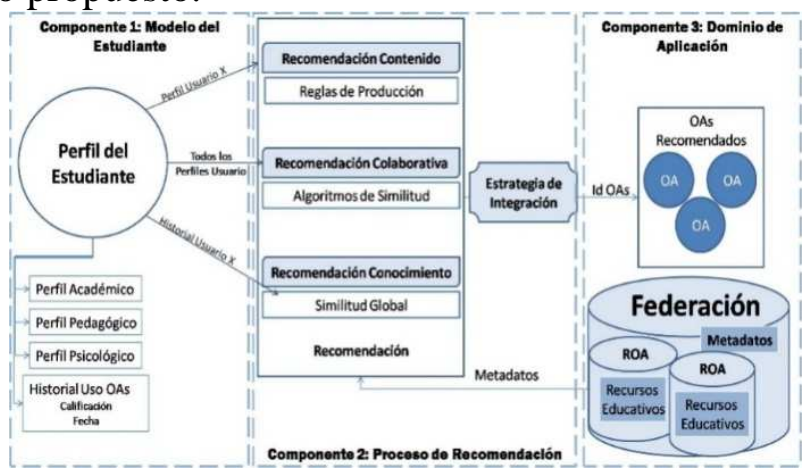

Figura 1. Modelo Integrado de Recomendación Propuesto

Componente 1: Modelo del Estudiante: se definen las características del estudiante para realizar el proceso de recomendación. Para este modelo se toma en cuenta las características del estudiante como su perfil académico, perfil pedagógico y finalmente el perfil psicopedagógico donde el estilo de aprendizaje es el factor más relevante para hacer las recomendaciones (Alonso et al., 1997).

Componente 2: Proceso de Recomendación: pertenecen las diferentes técnicas de recomendación que pueden ser utilizadas para entregar OA adaptados:

Recomendación por contenido: Esta recomendación está basada en el perfil del estudiante. En un trabajo preliminar (Rodríguez et al., 2012) se presenta el estudio donde se asocian los metadatos que utilizan los ROA con algunas características del perfil del estudiante obtenido explícitamente. En la cadena de búsqueda se incluyen la consulta de los valores para los metadatos Learning Resource Type, Interactivity Level, Intended end User Role, Context, Description y Language, los cuales se confrontan con el estilo de aprendizaje y se seleccionan aquellos OA que personalicen los resultados.

Recomendación colaborativa: La recomendación basada en filtrado colaborativo, pretende entregar OA a los estudiantes, buscando OA que le gustaron a usuarios similares. La similitud puede definirse como la representación numérica del grado de semejanza entre dos usuarios, basada en las preferencias sobre ítems comunes (Del Pino et al., 2011). Para hacer este proceso primero se busca un perfil similar a través de algún algoritmo de similitud, para este caso se seleccionó la distancia del coseno según el estudio presentado en (Rodríguez et al., 2014), enfocándose en 6 características del perfil del estudiante para encontrar la similitud de los usuarios. Recomienda OA del usuario similar que fueron evaluados positivamente y que fueron más recientemente utilizados.

Recomendación basada en conocimiento: Finalmente la recomendación basada en conocimiento, hace recomendaciones buscando OA similares a los que el usuario evaluó positivamente en el pasado. Para esto primero se realiza un proceso de filtrado de los OA evaluados con una calificación igual o superior a 4, en una escala de 1 a 5 , y se buscan OA similares, realizando búsquedas por los metadatos título, descripción y palabras clave de cada OA a recomendar con los OA evaluados utilizando la medida overlap.

Estrategia de Integración: Para que el modelo entregue una recomendación híbrida se deben definir los criterios para ordenar los resultados de las otras técnicas de recomendación, para ello se utilizan las intersecciones entre los resultados: en primer lugar entrega los $\mathrm{OA}$ que son comunes a los tres procesos de recomendación, luego 
continua con los OA comunes en dos de ellos y finalmente los OA que están sólo en una lista de recomendaciones.

Componente 3: Dominio de Aplicación: El modelo de recomendación híbrida puede ser aplicado a ROA y federaciones de ROA, que es lo que se prueba en este artículo.

\section{Método para la validación del sistema de recomendación híbrido}

Con el fin de verificar el correcto funcionamiento del sistema de recomendación y contrastar que los resultados sean relevantes para los usuarios se presenta un plan de pruebas, donde se puede concluir que un sistema de SR recomendación híbrido para entregar OA adaptados es una buena alternativa para apoyar al estudiante en el proceso de enseñanza-aprendizaje.

\subsection{Sistema Utilizado}

En un trabajo preliminar presentado (Rodríguez et al., 2013) que está en el marco de la tesis de maestría de Rodríguez, se presenta el modelo propuesto, es cual se utilizará para realizar las pruebas, el sistema está disponible en FROAC (http://froac.manizales.unal.edu.co/froac/).

\subsection{Pruebas a realizar}

Se aplicaran dos tipos de pruebas para realizar la validación del modelo de recomendación, la primera de ellas $M A E$ se realiza a los diferentes repositorios y federaciones; la segunda precisión, solo será aplicada a los OA recuperados de FROAC.

\subsubsection{Prueba MAE}

Error medio absoluto (Mean Absolute Error, MAE), es un método estadístico que permite medir la desviación de las recomendaciones predichas con los valores reales, a menos MAE mejor predicción del sistema de recomendación (Salazar y Ortega, 2006). En la ecuación 1 se presenta como se calcula esta medida.

$$
M A E=\frac{1}{N} \sum_{i=1}^{N}\left|x_{i}-\hat{x}_{i}\right|
$$

Donde $X_{i}$ es el valor esperado (de la recomendación) y $\hat{x}_{i}$ es el valor real (Calificación) y $\mathrm{N}$ es la cantidad de ítems a ser valorados.

El MAE será calculado para el resultado final de los SR Contenido, Colaborativo, Conocimiento e Híbrido, entonces el valor real es la calificación que da en promedio un conjunto de estudiantes a un $\mathrm{OA}$ en una escala de 1 a 5 , siendo 5 el valor máximo que representa la relevancia de ese objeto; por otro lado el valor esperado $\mathrm{X}_{\mathrm{i}}$ será calculado así para cada OA: para las recomendaciones por contenido, colaborativo y conocimiento si OA es recomendado tomará una calificación de 5 y sino tiene una de 0 . Para el resultado de la integración, es decir fue recomendado por las tres técnicas del sistemas de recomendación, se le dará una calificación esperada de 5, si el OA es el resultado de dos de las técnicas de recomendación del modelo su calificación será de 4 y si el OA solo fue recomendado por una técnica su calificación será de 3 .

\subsubsection{Precisión}

La segunda prueba es una métrica de decisión para calcular la calidad de las recomendaciones, se seleccionó la medida de precisión que es comúnmente utilizada para evaluar la recuperación de información. Según la ecuación 2.

La precisión será calculada, como el cociente del número de OA relevantes sobre el total de objetos entregados y la relevancia entendida como la importancia que tiene un OA 
para apoyar el proceso de aprendizaje de un estudiante especifico, adaptándose a sus preferencias y necesidades, la relevancia será determinada por los estudiantes que calificaron dicho factor.

$$
\text { Precision }=\frac{\text { OAs relevantes }}{\text { OAs relevantes }+ \text { OAs recuperados }}
$$

\subsection{Alcance}

Para la validación se seleccionaron los siguientes repositorios y federaciones según un estudio cienciométrico presentado en (Rodríguez M et al., 2012) y por su accesibilidad a los metadatos necesarios para la ejecución del proceso de recomendación. Ver tabla 1. En cada uno de ellos se realizó una búsqueda y se seleccionaron $10 \mathrm{OA}$, este valor es pequeño debido que un conjunto de estudiantes debe dar una calificación a cada OA.

Tabla 1. Repositorios y federaciones de repositorios de OA utilizados en las validaciones

\begin{tabular}{|l|l|l|l|}
\hline \multicolumn{2}{|c|}{ Repositorios } & \multicolumn{2}{c|}{ Federaciones } \\
\hline Nombre & Ubicación & Nombre & Ubicación \\
\hline Gateway & http://www.thegateway.org/ & FROAC & http://froac.manizales.unal.edu.co/froac/ \\
\hline Jorum & http://find.jorum.ac.uk/ & Ariadne & http://ariadne.cs.kuleuven.be/AriadneFinder/ \\
\hline Merlot & http://www.merlot.org/merlot/index.htm & Feb & http://feb.ufrgs.br/feb/ \\
\hline
\end{tabular}

\subsection{Usuarios}

Se seleccionó un grupo de estudiantes de Administración de Sistemas Informáticos de la Universidad Nacional de Colombia Sede Manizales, para que realizaran la revisión y la evaluación a cada uno de los OA presentados de cada repositorio. Los estudiantes se registraron en FROAC y realizaron el test de estilos de aprendizaje.

\section{Validación del Sistema de Recomendación Híbrido}

\subsection{MAE}

Para hacer el cálculo de la medida MAE, se realizó una búsqueda en el lenguaje original de cada repositorio y federación la palabra "algoritmos", se seleccionaron los primeros 10 OA y se entregaron al SR para que los procesará, se realizó el cálculo del MAE para cada uno de los SR y finalmente se evalúo el resultado de la integración de las recomendaciones (resultado del SR híbrido), por otro lado se le entregaron esos OA al conjunto de estudiantes para que evaluara en una escala de 1 a 5 el OA, bajo los criterios de si le parecía que el OA era relevante. Los resultados se muestran en la tabla 2 y la figura 3. Recordemos que entre más cercano al 0 este la medida mejor predicciones realizó.

Tabla 2. Resultados pruebas MAE

\begin{tabular}{|l|r|r|r|r|}
\hline \multicolumn{1}{|c|}{ ROA /Federación } & \multicolumn{1}{c|}{ SR Contenido } & SR Colaborativo & SR Conocimiento & \multicolumn{1}{c|}{ SR Hibrido } \\
\hline FROAC & 1,26 & 2,74 & 0,26 & 0,384615385 \\
\hline Ariadne & 0,34 & 1,34 & 1,34 & 0,2 \\
\hline Feb & 0,28 & 3,28 & 0,28 & 0,88 \\
\hline Merlot & 0,825 & 1,825 & 1,825 & 1,225 \\
\hline Gateway & 1,225 & 1,225 & 1,225 & 1,225 \\
\hline Jorum & 2,175 & 2,175 & 2,175 & 2,175 \\
\hline
\end{tabular}




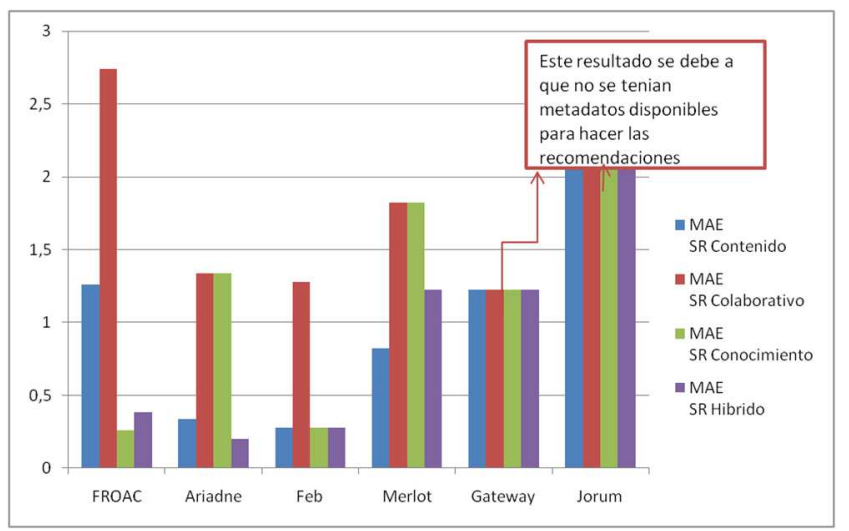

Figura 3. Resultados pruebas MAE

De los datos y la figura anterior se puede deducir que en la mayoría de los casos el SR híbrido entrega mejores recomendaciones que las otras técnicas, por lo cual se concluye que el uso de los SR Híbridos entregan OA adaptados a los estudiantes y que es válido utilizar el modelo propuesto en cualquier ROA y federación siempre y cuando este tenga los metadatos disponibles.

Para cada uno de los ROA y federaciones evaluadas se pueden concluir los siguientes aspectos tanto por el lado del SR como de la interacción con el usuario.

FROAC: la descarga de los OA hace difícil la visualización.

ARIADNE: hay muchos OA que no están disponibles, la descarga de los OA para su visualización hace tediosa su utilización. Por último no tienen disponibles los metadatos de la categoría educacional.

FEB: La federación de Brasil, aunque cosecha todos los metadatos no están disponibles los de la categoría educacional.

MERLOT: Al ser un ROA reconocido y con una cantidad considerable de OA, los primeros resultados de su búsqueda no eran los más relevantes.

GATEWAY: Este ROA no tenía disponibles los metadatos necesarios para aplicar las técnicas de recomendación, es por esto que los resultados son iguales para los 4 procesos; lo mismo ocurre con JORUM.

\subsection{Precisión}

Se realizó una búsqueda en FROAC con la palabra "Algoritmos" y la palabra "programación", para un tipo de usuario con un estilo de aprendizaje: "Visual-Global", las preferencias son la programación, análisis y diseño de algoritmos, además estos estudiantes evaluaron positivamente (evaluación $>=4$ ) algunos OA referentes a la programación exhaustiva. Al hacer la búsqueda se encontraron los siguientes OA presentados por identificación en la tabla 3 y 4 . En la última fila de la tabla se puede observar los resultados de la medida de precisión para cada una de las técnicas de recomendación.

Con los resultados obtenidos se puede concluir que el SR Híbrido, entrega los OA adaptados al perfil del estudiante y además entrega OA relevantes para apoyar el proceso de enseñanza aprendizaje. Además se puede concluir que la estrategia de integración es adecuada y entrega OA relevantes. Se deben realizar nuevas pruebas con otros OA para continuar con esta validación, debido que solo se realizó con dos palabras. 
Tabla 3. Resultados de la búsqueda "Algoritmos" y "Programación" identificación de los $\mathrm{OA}$

\begin{tabular}{|c|c|c|c|c|}
\hline Estudiante & \multicolumn{4}{|c|}{ Sistema de Recomendación Híbrido Propuesto } \\
\hline OAs Relevantes & SR Contenido & SR Colaborativo & SR Conocimiento & SR Hibrido \\
\hline 274 & 274 & & 274 & \\
\hline 276 & 276 & & 276 & \\
\hline 277 & 277 & & 277 & \\
\hline 275 & 275 & & 275 & \\
\hline 272 & 272 & & 272 & \\
\hline 269 & 269 & 269 & 269 & 269 \\
\hline 271 & 271 & 271 & 271 & 271 \\
\hline & 189 & & 189 & \\
\hline & 191 & & & \\
\hline 334 & 334 & 334 & 334 & 334 \\
\hline & 194 & & & \\
\hline & 197 & & 197 & \\
\hline & 198 & & & \\
\hline 273 & 273 & & 273 & \\
\hline & 196 & & & \\
\hline Precisión & 0,529411765 & 1 & 0,818181818 & 1 \\
\hline
\end{tabular}

\begin{tabular}{|c|c|c|c|c|}
\hline Estudiante & \multicolumn{4}{|c|}{ Sistema de Recomendación Híbrido Propuesto } \\
\hline OAs Relevantes & SR Contenido & SR Colaborativo & SR Conocimiento & SR Hibrido \\
\hline 262 & 262 & & & \\
\hline 266 & 266 & & & \\
\hline 260 & 260 & & & \\
\hline 259 & 259 & & & \\
\hline 264 & 264 & & & \\
\hline 267 & 267 & & & \\
\hline & 209 & & & \\
\hline & 412 & & & \\
\hline & $\begin{array}{l}411 \\
411\end{array}$ & & & \\
\hline & $\begin{array}{l}410 \\
408\end{array}$ & & & \\
\hline & 281 & & & \\
\hline 258 & $\begin{array}{l}413 \\
258\end{array}$ & & & \\
\hline 269 & 269 & 26 & & 269 \\
\hline 235 & 235 & & & \\
\hline 256 & 256 & & 256 & 256 \\
\hline Precisión & 0,555555556 & 1 & 1 & 1 \\
\hline
\end{tabular}

\section{Conclusiones}

En el presente trabajo se validó el SR Híbrido de Objetos de aprendizaje presentado en (Rodríguez et al., 2013). Dicho sistema es accedido vía web, mediante la federación de repositorios de objetos de aprendizaje Colombia FROAC (http://froac.manizales.unal.edu.co/froac/) y permite a los usuarios registrados acceder a OA adaptados a sus características y preferencias. Se seleccionaron e implementaron dos pruebas de validación para este sistema el MAE -error cuadrático medio- (para validar el comportamiento de los SR que conforman el modelo en diferentes ROA y federaciones de ROA) y por otro lado la medida de precisión para evaluar la relevancia de los OA recomendados; consiguiendo entonces, con la primera prueba que el sistema propuesto se puede aplicar a cualquier repositorio o federación de repositorios de OA que tengan disponibles sus metadatos y que es relevante utilizar SR híbridos que integren los resultados de las otras técnicas de recomendación. Y con la segunda prueba se concluyó que el SR entrega OA adaptados al perfil del estudiante que son relevantes para su proceso de enseñanza - aprendizaje. Como trabajo futuro se propone hacer nuevas pruebas teniendo en cuenta el orden en que son recomendados los OA.

\section{Agradecimientos}

El trabajo de investigación presentado en este artículo fue financiado parcialmente por el proyecto "Mejoramiento de la capacidad académica, visibilidad, contacto e interacción con la comunidad Nacional e Internacional del grupo de investigación en Inteligencia Artificial de la Universidad Nacional" con código 14163 financiada por la VRI en el programa de Fortalecimiento de Grupos de Investigación y Creación Artística de la Universidad Nacional de Colombia 2011-2012.

\section{Bibliografía}

Al-Khalifa, H. S. (2008). Building an Arabic learning object repository with an ad hoc recommendation engine. Proceedings of the 10th International Conference on Information Integration and Web-based Applications \& Services - iiWAS '08, 390. doi:10.1145/1497308.1497378 
Alonso, C., Gallego, D., \& Honey, P. (1997). Los Estilos de Aprendizaje. Procedimientos de diagnostico y mejora. Bilbao.

Burke, R. (2002). Hybrid Recommender Systems: Survey and Experiments. User Modeling and User-Adapted Interaction, 12(4), 331-370.

Burke, R. (2007). Hybrid web recommender systems. The adaptive web, 4321, 377408. Retrieved from http://link.springer.com/chapter/10.1007/978-3-540-720799_12

Casali, A., Gerling, V., Deco, C., \& Bender, C. (2011). Sistema inteligente para la recomendación de objetos de aprendizaje. Revista Generación Digital, 9(1), 88-95.

Cazella, S. C., Reategui, E. B., \& Nunes, M. A. (2010). A Ciência da Opinião: Estado da arte em Sistemas de Recomendação. JAI: Jornada de Atualização em Informática da SBC. Rio de Janeiro, RJ: PUC Rio, 161-216.

Chesani, F. (2002). Recommmendation Systems. Corso di laurea in Ingegneria Informatica, 1-32.

Del Pino, J., Salazar, G., \& Cedeño, V. (2011). Adaptación de un Recomendador de Filtro Colaborativo Basado en el Usuario para la Creación de un Recomendador de Materias de Pregrado Basado en el Historial Académico de los Estudiantes. Revista Tecnológica ESPOL - RTE, 24(2), 29-34. Retrieved from http://learningobjects2006.espol.edu.ec/index.php/tecnologica/article/view/85

Downes, S. (2001). Learning objects: resources for distance education worldwide. The International Review of Research in Open and Distance Learning, 2(1), 1-17. Retrieved from http://www.doaj.org/doaj?func=fulltext\&aId=203793

Edwards, D., Rai, S., Phillips, R., \& Fung, L. C. C. (2007). A Framework for Interoperable Learning Objects for E-Learning. In Learning Objects and Instructional Design. Santa Rosa, California: Informing Science Press (pp. 437469).

García, N. M., \& Montoyo, A. (2008). Recomendación de objetos de aprendizaje almacenados en repositorios lor@ server según las preferencias del usuario . Universidad Agraria de La Habana y Universidad de Alicante.

Gil, A. B., \& García, F. (2007). Un Sistema Multiagente de Recuperación de Objetos de Aprendizaje con Atributos de Contexto. ZOCO'07 / CAEPIA.

Hdioud, F., Frikh, B., \& Ouhbi, B. (2012). A comparison study of some algorithms in Recommender Systems. Information Science and Technology (CIST), Colloquium, 130-135. Retrieved from http://ieeexplore.ieee.org/xpls/abs_all.jsp?arnumber=6388076

Kahn, R., \& Wilensky, R. (2006). A framework for distributed digital object services. International Journal on Digital Libraries, 6(2), 115-123. doi:10.1007/s00799005-0128-X

Learning Technology Standards Committee. (2002). IEEE Standard for Learning Object Metadata. Institute of Electrical and Electronics Engineers, New York.

Li, J. Z. (2010). Quality, Evaluation and Recommendation for Learning Object. International Conference on Educational and Information Technology, (Iceit), 533-537.

Mayorga, J. I., Celorrio, C., Lorenzo, E. J., Vélez, J., Barros, B., \& Verdejo, M. F. (2007). Comunidades Virtuales de Aprendizaje Colaborativo: de los Metadatos a la Semántica. Revista Iberoamericana de Inteligencia Artificial, 11(33), 47-60.

Mizhquero, K. (2009). Análisis , Diseño e Implementación de un Sistema Adaptivo de Recomendación de Información Basado en Mashups. Revista Tecnológica ESPOL. 
Niemann, K., Scheffel, M., Friedrich, M., Kirschenmann, U., Schmitz, H., \& Wolpers, M. (2010). Usage-based Object Similarity. Journal of Universal Computer Science, , 16(16), 2272-2290.

Ouyang, Y., \& Zhu, M. (2008). eLORM: learning object relationship mining-based repository. Online Information Review, 32(2), 254-265. doi:10.1108/14684520810879863

Peña, C. I., Marzo, J., De la Rosa, J. L., \& Fabregat, R. (2002). Un sistema de tutoría inteligente adaptativo considerando estilos de aprendizaje. Universidad de Girona, España.

Primo, T. T., Vicari, R. M., \& Marques, J. (2009). Rumo ao Uso de Metadados Educacionais em Sistemas de Recomendação.

Rodríguez M, P. A., Isaza, G., \& Duque, N. D. (2012). Búsqueda personalizada en Repositorios de Objetos de Aprendizaje a partir del perfil del estudiante. Revista Avances Investigación en Ingeniería, 9(1), 73-83.

Rodríguez, P. A., Duque, N. D., \& Ovalle, D. A. (2013). Modelo Integrado de Recomendación de Objetos de Aprendizaje. In CAVA 2013 - V Congreso Internacional de Ambientes Virtuales de Aprendizaje Adaptativos y Accesibles. (pp. 1-6).

Rodríguez, P. A., Moreno, J., Duque, N. D., Ovalle, D., \& Silveira, R. (2014). Un modelo para la composición semiautomática de contenido educativo desde repositorios abiertos de objetos de aprendizaje A model for the semi-automatic composition of educational content from open repositories of learning objects. Revista Electrónica de Investigación Educativa (REDIE)., 16(1).

Sabitha, a S., Mehrotra, D., \& Bansal, A. (2012). Quality metrics a quanta for retrieving learning object by clustering techniques. 2012 Second International Conference on Digital Information and Communication Technology and it's Applications (DICTAP), 428-433. doi:10.1109/DICTAP.2012.6215396

Sanjuán, O., Torres, E., Castán, H., Gonzalez, R., Pelayo, C., \& Rodriguez, L. (2009). Viabilidad de la aplicación de Sistemas de Recomendación a entornos de elearning. Universidad de Oviedo, España.

Sicilia, M.-Á., García-Barriocanal, E., Sánchez-Alonso, S., \& Cechinel, C. (2010). Exploring user-based recommender results in large learning object repositories: the case of MERLOT. Procedia Computer Science, 1(2), 2859-2864. doi:10.1016/j.procs.2010.08.011

Tabares, V., Rodríguez, P., Duque, N., \& Moreno, J. (2012). Modelo Integral de Federación de Objetos de Aprendizaje en Colombia-más que búsquedas centralizadas. Séptima Conferencia Latinoamericana de Objetos y Tecnologías de Aprendizaje, 410-418. Retrieved from http://laclo.org/papers/index.php/laclo/article/view/40

Vicari, R. M., Ribeiro, A., Marques, J., Rizzon Santos, E., Primo, T., \& Bez, M. (2009). Brazilian Proposal for Agent-Based Learning Objects Metadata Standard - OBAA. Universidad Federal do Rio Grande do Sul.

Wang, T. I., Tsai, K. H., Lee, M. C., \& Chiu, T. K. (2007). Personalized Learning Objects Recommendation based on the Semantic Aware Discovery and the Learner Preference Pattern. Educational Technology \& Society, 10(3), 84-105.

Wiley, D. A. (2001). Connecting learning objects to instructional design theory: A definition, a metaphor, and a taxonomy. The Instructional Use of Learning Objects. Agency for Instructional Technology, 2830(435), 1-35. 